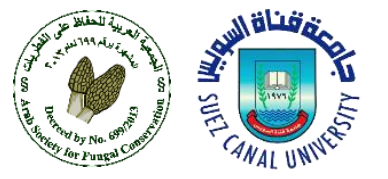

Contents lists available at Egyptian Knowledge Bank

Microbial Biosystems

Journal homepage: http://mb.journals.ekb.eg/

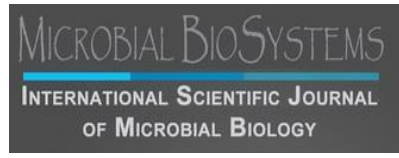

\title{
Molecular dual actions of hsa-miRNA and v-miRNA in oncogenic EBV
}

\section{Rezhna Kheder Ali', Sazan Qader Maulud ${ }^{1,2}$, Paywast Jamal Jalal ${ }^{3}$, Jivan Qasim Ahmed $^{4 *}$}

${ }^{1}$ Department of Biology, College of Education, Salahaddin University-Erbil, Kurdistan Region, Iraq

${ }^{2}$ Department of Medical Laboratory Science, College of Science, Knowledge University, Kirkuk, Iraq

${ }^{3}$ Department of Biology, College of Science, University of Sulaimani, Kurdistan Region, Iraq

${ }^{4}$ Department of Pathology and Microbiology, University of Duhok, Kurdistan Region, Iraq

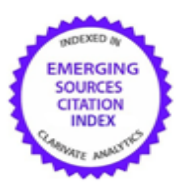

\section{ARTICLE INFO \\ Article history \\ Received 21 July 2021 \\ Received revised 13 August 2021 \\ Accepted 13 August 2021 \\ Avaliable online 21 August 2021 \\ (C) Ali et al., 2021}

Corresponding Editor:

T. A. Mohammed

Y. El-Maradny

Keywords

EBV

Oncovirus

viral miRNA

host miRNA

\begin{abstract}
Viruses are one of the main reasons that cause healthy cells to proliferate and become cancerous. Several viruses have been identified as causative factors for various forms of cancer. Tumor occurrence can be caused by viral oncoprotein activity, persistent infection or inflammation. The molecular process is still complicated to be understood. In recent decades, Homo sapiens cell microRNA (hsa-miRNA) has been discovered by small non-coding RNAs that affect post-transcriptional gene expression. hsamiRNA is a key control factor for several key biological processes and has a much greater impact on the target gene group. even though they occupy a small part of the genome, they play a great role in the development of cancer. Several viruses produce this tiny RNA, which can regulate their gene expression or affect the host's gene expression. A new hypothesis is that Epstein-Barr virus (EBV) is the first cancer causing virus that is found to produce microRNAs (v-miRNAs). In addition, evidence shows that miRNA encoded by EBV contributes to the occurrence and progression of EBV-related malignancies. Generally, these compounds reduce messenger RNA (mRNA) instability, such as genes that regulate tumorigenesis mechanisms like inflammation, cell cycle control, stress response, differentiation, apoptosis, invasion, and immune pathways. Therefore, EBV-miRNAs are important in the complex interaction between host, virus and EBV tumorigenesis. In terms of malignant tumors, the combinatorial process behind EBV-miRNA still needs further study. In this article, we will introduce EBV-miRNA, including the cellular processes affected by the virus, and their ability to promote cancer.
\end{abstract}




\section{Introduction}

Nowadays, scientific data support the hypothesis that viruses have a place in human malignancies (Gallo et al. 2020). Recently, viruses are one of the main causes of normal cell proliferation and cancer (Pfeffer and Voinnet, 2006). According to the latest IARC (International Agency for Research on Cancer) report, chronic infections, primarily viral infections, were responsible for $13 \%$ of newly diagnosed malignancies globally in 2018 (Wang et al. 2019). Oncogenic viruses cause and maintain a long-term infection through evading the immune response, which is consistent with the carcinogenic mechanism. Most oncogenic viruses have mechanisms that allow them to be equally divided into daughter cells throughout cell division, enabling their genome to be maintained in host cells during proliferation. Then, the immortalization of cells is caused by the virus, either directly or indirectly. The implementation process involves deregulation of cellular oncogene expression/tumor suppressor genes influenced by viral genome incorporation in the host genome (examples of retroviruses are: human papillomavirus and hepatitis B virus ), or the expression of cancer causing virus (e.g herpesvirus) induce damage to DNA and host cells by inhibiting the basic mechanisms of genome constancy and cell cycle. Indirect modification processes include immune cells and tissue damage caused by ongoing inflammatory processes or immunosuppression caused by viral infections, resulting in suppression of the anti-tumor surveillance system (Vojtechova and Tachezy, 2018). Several viruses have been identified as pathogens of specific types of cancer (Pfeffer and Voinnet, 2006). Viruses that are Categorized as oncogenic viruses, include Epstein-Barr virus, human papillomavirus, hepatitis C virus, hepatitis B virus, human herpesvirus 8 or polyomavirus, and trans-regulatory retroviruses, such as (HIV or HTLV-1) (Vojtechova and Tachezy, 2018). Furthermore, the incidence of malignant tumors caused by oncogenic viruses have increased, indicating that there is profound interaction between the virus and the immune system in certain conditions of immunosuppressed people. Innate immune signals have many key effector proteins, including the cyclin-dependent kinase blockers p21 and $\mathrm{p} 53$, and related tumor suppressor signals (Gallo et al. 2020).

Several short forms of RNA that affect several biological processes in cells have just been discovered. One of these includes miRNAs (Louten et al. 2015), about 19 to 25 bases in length, which has an essential role in the regulation of post-transcriptional gene expression (Palmero et al. 2011 and Tan et al. 2018). Thus, they have an impact on important activities such as proliferation, apoptosis as well as lipid metabolism in cell biology by attaching them to mRNA. miRNAs induce blockade of mRNA translation, thereby reducing expression levels of recipient genes (Židovec Lepej et al. 2020). Cancerrelated miRNA, hsa-miRNA and v-miRNA biosynthetic pathways are shown in (Fig. 1). In addition, hsa-miRNA can induce immune invisibility of infected cells (Gallo et al. 2020). In 1993, in Caenorhabditis elegans, the first miRNA named lineage-deficient-4 (Lin-4) was found to be involved in the management of larval development. Another miRNA (let-7) was also found in Caenorhabditis elegans, in 2000 (Palmero et al. 2011). Small repeats of single-stranded RNA exhibit an inhibitory effect on complementary mRNA. The study of miRNA provides knowledge about the origins of diseases, therapy and surveillance, such as viral diseases (Louten et al. 2015). in recent decades have seen an increasing number of research done on human cancer, which have uncovered cellular miRNAs with their invaluable possibilities as diagnostic and prognostic biomarker (Gallo et al. 2020). Much of the research on v-miRNAs, particularly in carcinogenesis, has focused on v-miRNAs in the EpsteinBarr virus, the herpes virus associated with Kaposi's sarcoma, and the human papillomavirus, hepatitis B virus and hepatitis C virus (Židovec Lepej et al. 2020).

\section{Epidemiology of the EBV virus that causes cancer}

Epidemiological and serological studies show that Epstein-Barr virus infection has an original relationship with native Barkit's lymphoma and other neoplasms (Chang et al. 2017). Epstein Barr-associated epithelial malignancies, including nasopharyngeal cancer (NPC), in Southeast Asia, southern China, where more than $97 \%$ of NPC patients are EBV-positive (Cohen et al. 2011). Several risk factors are suggested, including host immunity-related genes, human leukocyte antigen, and nutrition (Liebowitz, 1994). In addition, genetic or postmortem abnormalities in host cells lead to the formation and progression of nasopharyngeal cancer (Kanda et al. 2019). Recently, there has been seven different viral infection that were involved in differnet human cancer development, including EBV, Kaposi sarcoma-associated herpesvirus (KSHV), high-risk Human papilloma virus (HPV), Hepatitis B virus (HBV), Hepatitis C virus (HCV), Merkel cell polyomavirus (MCPV), and Human T-lymphotropic virus type 1 (HTLV1), infection is thought to contribute to $20 \%$ of all cancer development globally (Morales-Sánchez and Fuentes-Pananá, 2014). Studies show that if these viruses are eradicated in developing countries, the cancer incidence would be reduced by $23.6 \%$ and in developed countries by $7.7 \%$, 
preventing 1.5 million $(390,000)$ cancer cases per year (Parkin, 2006). Oncoviruses are considered the second

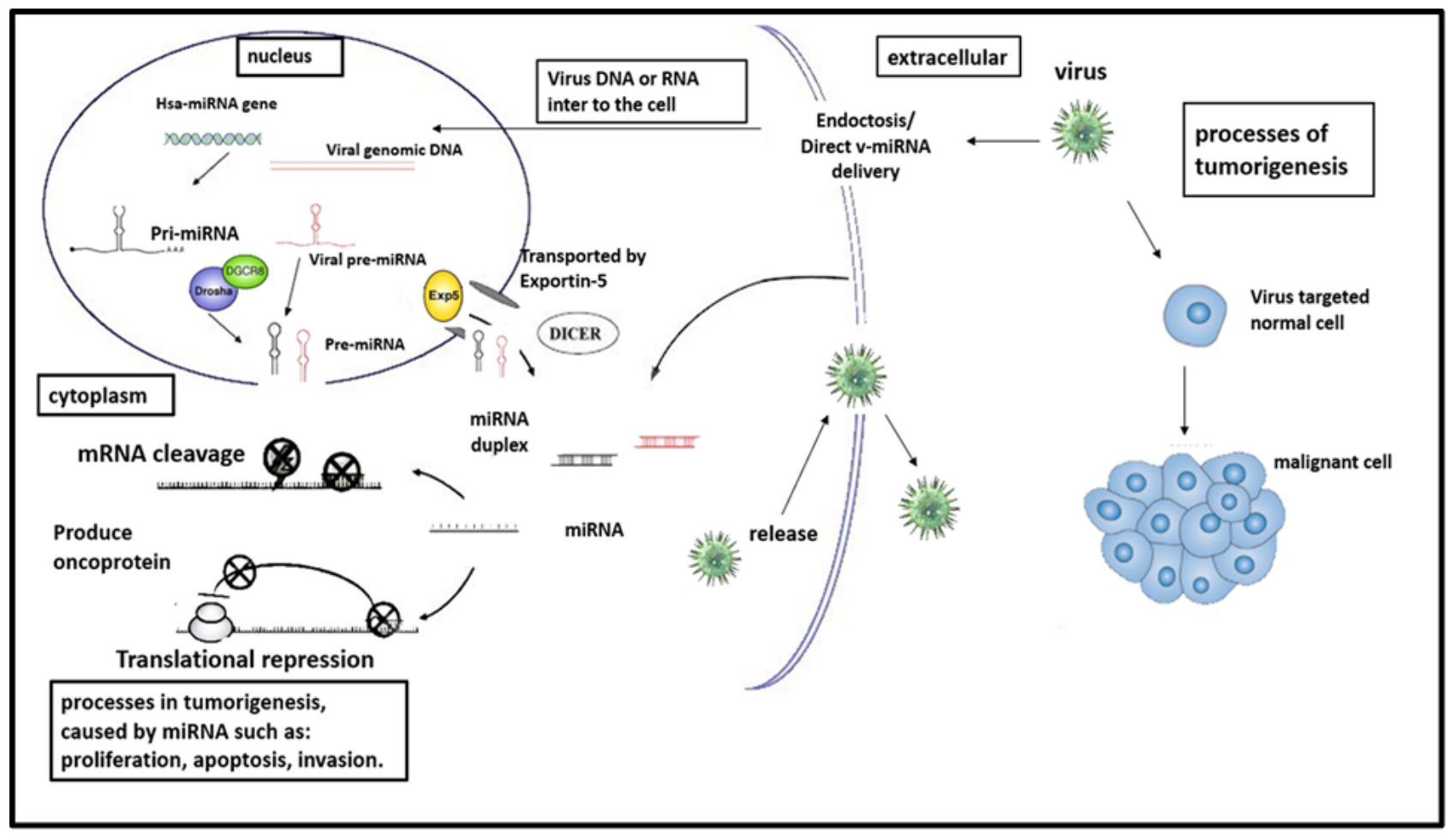

Fig 1. The mechanism of hsa-miRNA and v-miRNA biogenesis, and associated with cancer development.

most important risk factor for cancer growth in humans after tobacco use (Rwazavian, 2011). Many viruses are now demonstrated to be carcinogenic to the human, and the first oncovirology studies of the first century ended with the award of the Harald Chuerhausen Nobel Prize in Medicine to Human papillomavirus (HPV) as a causative agent of cervical cancer (Morales-Sánchez and FuentesPananá, 2014).

The oncoviruses can induce cancers through shared natural host cell targets and pathways. Both EBV and KSHV are major DNA viruses, and they can induce solid tissue cancers as well as lymphoid tissue tumors (McLaughlin-Drubin and Munger, 2008). Human papillomavirus (HPV) and Merkel cell polyomavirus (MCPyV) have fewer DNA genomes than EBV and KSHV. These small DNA oncoviruses utilize intermediate multifunctional oncoproteins to induce carcinogenesis (Feng et al. 2008). Human oncoviruses have several of shared properties that can cause human cancer. After transmission and establishing infection, they can end up in persistent infection without noted symptoms that lasts for many years. Subsequently, these viruses appoint cell mechanisms for viral replication and immune evasion. They interfere with the cellular signaling system that limits cell cycle progression and cell death to promote their replication (Krump and You, 2018). Viral oncogenic processes usually involve genomic instabilities production, rise in involvement in cell proliferation, apoptosis resistance, modification of DNA repair processes as well as modifications in cell polarity that sometimes coincide with antiviral immune responses and evasion mechanisms (Morales-Sánchez and FuentesPananá, 2014). Some of the characteristics of the seven oncoviruses are similar to most other viruses, it's important to find and identify the required feature to alter the mechanisms of cellular environment. (Krump and You, 2018). The usual mechanisms used by oncogenic viruses, include $\mathrm{p} 53$ and $\mathrm{pRb}$ inactivation as well as other rise in propagation and survive of the targets, genome instability, telomer shortening interfere, cell polarity interference and v-miRNAs (Morales-Sánchez and Fuentes- Panana, 2014).

\section{Mechanism of v-miRNA in oncogenesis}

Mechanisms of oncogenic v-miRNA non-coding RNA molecules that contribute to cell transformation and regulate most gene expression have recently been presented. MiRNAs inhibit the translation of mRNAs, mainly according to the complementarity of their base pairs by translation regulation. Almost all malignancies have altered hsa-miRNA (Morales-Sánchez and FuentesPananá, 2014). The first virus to be reported as a viral coding miRNA (v-miRNA) was Epstein Barr virus (EBV), which is classified as a DNA virus in the 
herpesvirus family. It was detected in Burkitt lymphoma (BL) cells firstly, when the first infection of EBV is most common in children, and thereafter EBV remains in latent form often in resting memory B-cells and less commonly in T-cells, NK-cells, or epithelial cells. In addition, to Burkitt lymphoma, Epstein-Barr virus is a proliferative disease of Hodgkin lymphoma or post-transplant lymphoma through epithelial malignancies including $\mathrm{T}$ cell lymphoproliferative disorders or gastric cancer (Pavlovic et al. 2016). Impaired regulation of hsa-miRNA expression found in some tumors in humans, such as EBV-related malignancies. The expression of hsa-miRNA is down regulated after EBV infection of primary B cells (Krump and You, 2018).

Many Epstein-Barr virus sequences have been cloned many times, and studies of the genomic sequence of cloned RNA suggest a unique convolution of the miRNA gene (Pfeffer et al. 2004). EBV miRNAs can regulate cell proliferation or apoptosis, silence tumor suppressors, invasion as well as metastasis and avoid immune destruction are shown in (Fig. 2), that is contributed to EBV-related malignant carcinogenesis (Wang et al. 2019). EBV miRNAs are derived from five separate double-stranded RNA (dsRNA) precursors in clusters at both locations in the EBV genome (Pfeffer et al. 2004), with these five v-miRNAs in the periphery that are encoded in two located clusters; BHRF1 genes (miRBHRF1-1, miR-BHRF1-2 and miR-BHRF1-3) and BART predecessors (miR-BART1 and miR-BART2) (Krump and You, 2018). The Epstein-Barr virus currently uses the miRbase database (Vojtechova and Tachezy, 2018) to code 25 pre-miRNAs that produce at least 44 mature miRNAs. A diagram of the EBV genome encoding some potential miRNA products is shown in (Fig. 3). These vmiRNAs are capable of block apoptosis, as well as targeting cellular tumorigenesis, including (PUMA, Bin, TOMM22 and WIF1 genes) (Morales-Sánchez and Fuentes-Pananá, 2014b).

An advantage of viruses encoding miRNAs including Epstein Barr virus and all other viruses encoding miRNAs, is their capability of either host and viral gene expression regulation without viral proteins which facilitate the immune evasion of the infected cells (Vojtechova and Tachezy, 2018). In all latency types, viral BART miRNAs are limited to expression latency types 1 and 2. The BHRF1 miRNA is widely expressed in latent type III cells involving lytical infective cells, including B lymphoma cells. It is detected in latent type 1 and 2 cells. A promoter that is an important feature of its transcription is the expression change between BART and BHRF1 miRNAs (Wang et al. 2019). v-miRNA is expressed in different ways in many types of cancer. Many studies have previously been executed to show the expression of EBV-miRNAs in malignancies (Cosmopoulos et al. 2009). BART miRNAs (miRBART16, miR-BART17-5p, miR-BART1-5p) help transform cells by targeting the latent membrane protein 1 (LMP1) protein, the viral progenitor. MiR-BART22 regulates latent membrane protein 2 (LMP2A) translation in nasopharyngeal cancer, allowing EBV-infected cells to evade host immune surveillance. EBV uses miRNAs to control indirect conversion between the lysis phase and the incubation period For example miR-BART2 regulates the BALF5 gene DNA polymerase, contributing to the replication of the viral genome during the lysis phase and promoting the latent phase of the virus (Barth et al., 2008). miR-BART6-5p regulates viral replication and latency by eliminating the oncogenic EBV nuclear antigen 2 (EBNA2) virus (Iizasa et al. 2010).

MiRNAs from Epstein Barr virus can control the translation of hsa-mRNA and thus may influence the pathogenesis of malignancies. Negative regulation of p53 translation is through MiR-BART5-5p and miR-BART19$5 \mathrm{p}$, predicting that the target gene of p53 is a revised BART miRNA (e.g., miR-BART3 and miR-BART5) (Choy et al. 2008). The mechanism of action of human oncogenic viruses and how oncogenic viruses influence miRNA and target host gene expression in the control of their coded array gene expression is illustrated in Fig. (4).

Transformation mechanisms into growth factor $\beta$ (TGF- $\beta$ ) and Wnt signaling pathways influence apoptosis and transformation of nasopharyngeal cancer, as well as the effect of EBV-miRNA on the development of irradiated lymphatics. Abnormally produced BARTmiRNA may affect genes involved in the regulation of cell cycle, apoptosis and Wnt signaling in infected B cells. In particular, many $\mathrm{v}$-miRNAs are coordinated to hsa-miRNAs, including oncogenic miRNAs (cluster mir17-92) and immunoregulatory miRNAs (miR-142-3p and miR-155). Epstein-Barr virus-infected lymphoblastic cell line (LCL) identifies v-miRNA target species. The results showed that EBV-miRNA is related to basic immune cell proliferation and survival in many cellular mechanisms. miR-BART7 has the ability to increase the proliferation of nasopharyngeal cancer cells under the control of oncogenic pathways such as TGF signaling (Wang et al. 2019).

Control of tumor suppression is one of the processes underlying the carcinogenicity of EBV miRNAs. For example, miR-BART10 can interfere with b-transducin repeat containing E3 ubiquitin protein ligase (b-TrCP) from expression (Zeng et al. 2014). This down regulation caused by miR-BART10 could result in reduction in degradation of its substrates, including bcatenin and Snail, therefore enhance development of NPC. miR-BART7-3p is found to be up regulated in NPC, 
that might enhance the cancer growth. The mechanism is through miR-BART7-3p stimulating the phosphatase and tensin homolog (PTEN)/phosphoinositide 3-kinase $(\mathrm{PI} 3 \mathrm{~K}) /$ protein kinase $\mathrm{B}$ (Akt) pathway and induction of oncogenic transcription factors $\mathrm{c}-\mathrm{Myc}$ and c-Jun expression. miR-BART3 induces NPC to grow and transform through down regulation of the tumor suppressor deleted in cancer 1 (DICE1), gastric carcinoma
(GC) cell proliferation is induced by miR-BART19-3p. miR-BART6- 3p and miR-142 reduce the interleukin-6 receptor (IL-6R) expression and phosphatase and tensin homologue (PTEN), whereas miRNA-142 promotes the proliferation of Epstein-Barr virus- infected Barkit lymphoma (BL) cells (Table 1) (Zhou et al. 2016).

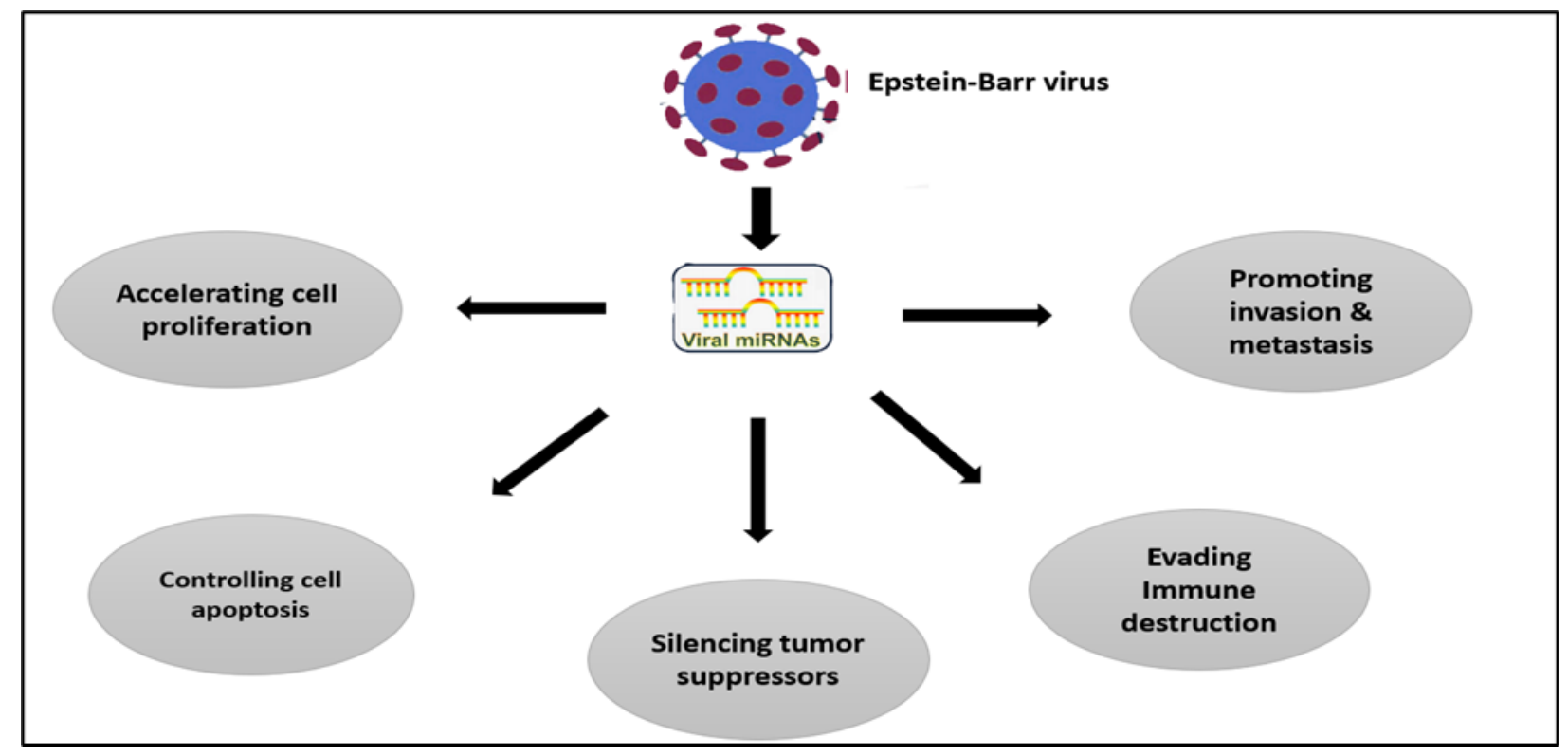

Fig 2. Mechanism of EBV on cancer development.

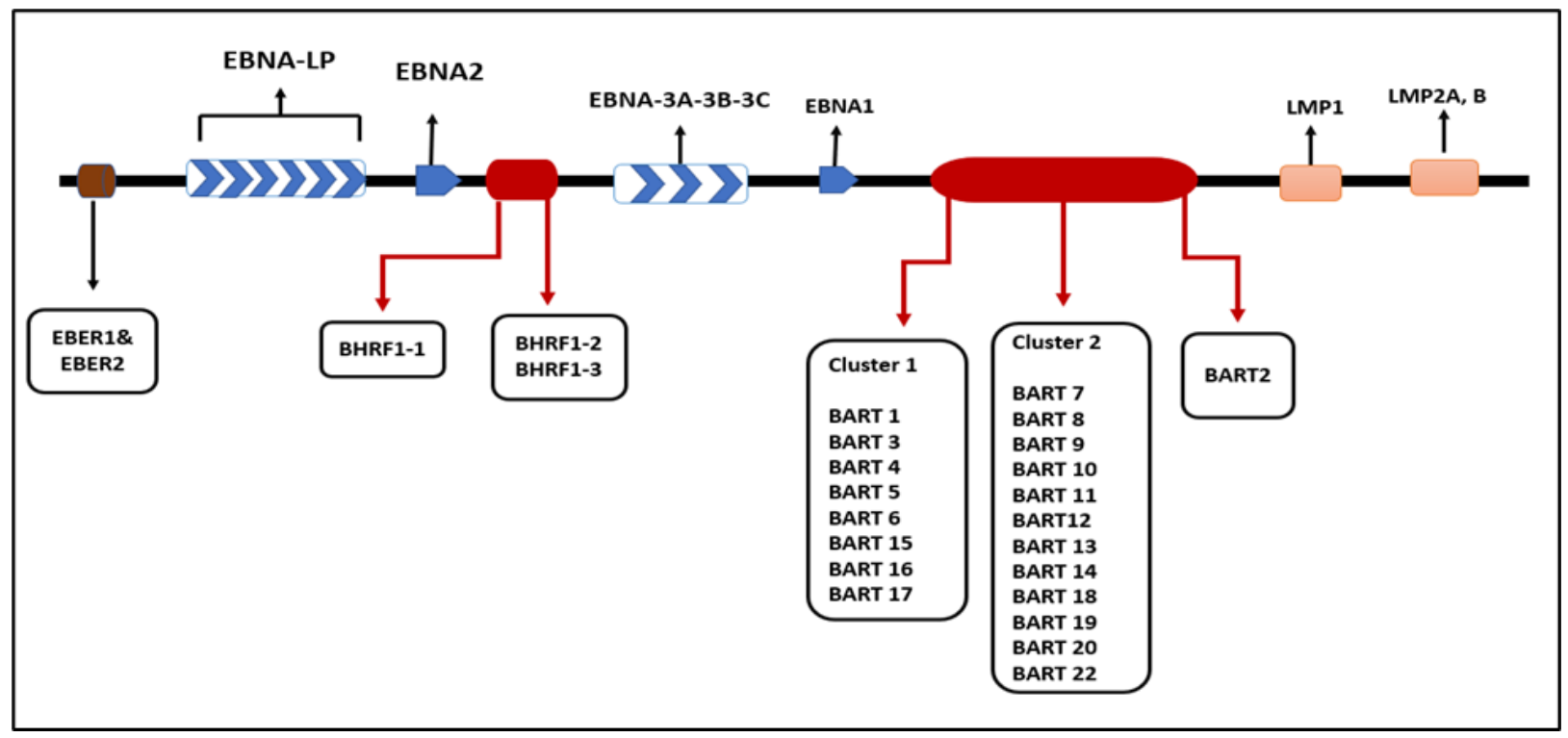

Fig 3. The structure of the EBV genome encoding potential production. Red boxes show miRNA code regions. BamHIRight Script (BART); BamHI engraves the right open reading frame 1 (BHRF1) of H. Encodes EBV-small molecule RNA (EBER). It encodes EBV-nuclear antigen 1 (EBNA1). It encodes EBV-nuclear antigen 2 (EBNA2). EBV-encoded nuclear antigen 3(EBNA3A, 3B, 3C); It encodes the EBV-nuclear leader protein (EBNA-LP). Latent membrane protein 1 (LMP 1). Latent membrane protein 2 (LMP2). 
The Epstein-Barr virus miRNAs also affects the translation of hsa-mRNA, which may involve in development of cancer. Therefore, miR-BART19-5p and miR-BART5-5p down regulate the p53 up regulatory mediator of cellular apoptotic process, which is a direct promoting aspect of programmed cell death, and are much lower in Epstein-Barr virus infected cells (Vojtechova and Tachezy, 2018). On the other hand, EBV miRNAs can limit the cancer cells growth by regulating proteins of apoptosis suppressor proteins. For example, miRBART15-3p suppresses gastric cancer (GC) cell growth and accelerates cell death through decreasing the level of the BIR repeat-containing ubiquitin-conjugating enzyme (BRUCE). However, miR-BART15-3p significantly reduce the expression of protein 1-bound anti-apoptotic tax-1 in gastric cancer (GC) cells that results in programed cell death. Altogether, EBV miRNAs are proficient in controlling cancer cell propagation by many pathways, including tumor suppressor gene silencing, the viral oncogenic proteins modulation, and establishing a tumor-promoting milieu. This allows v-miRNAs to affect many genes involved in various cellular processes. As a result, V-miRNAs and their target genes form a complex regulatory network throughout the development of cancer (Wang et al. 2019)

On the other hand, HHV-8 (Human-herpesvirus 8) which is also called Kaposi's sarcoma-related herpes virus because it triggers Kaposi's sarcoma (KS), a disease that induces vascular system and lymphatic endothelial cell proliferation. The Kaposi's sarcoma-related herpesvirus encrypts the pre-miRNAs of 12 viruses and eventually develops into 25 mature miRNAs. All v-miRNA genes are grouped and regulated by potential Kaposi promoters, except for miR-K10 and miR-K12, which are located on open-reading frame and 30 termini of the Kaposi genome respectively. Most of the pre-miRNA genes are introns and are contained within the arrangement of the Kaposi open reading frame. It is important to emphasize that the incubation period of the virus is necessary for the development of tumors. Kaposi's sarcoma-associated herpesvirus miRNAs regulate viral replication and thus function in viral life cycle management by targeting important viral genes directly and cellular genes involved in indirect tumorigenesis.

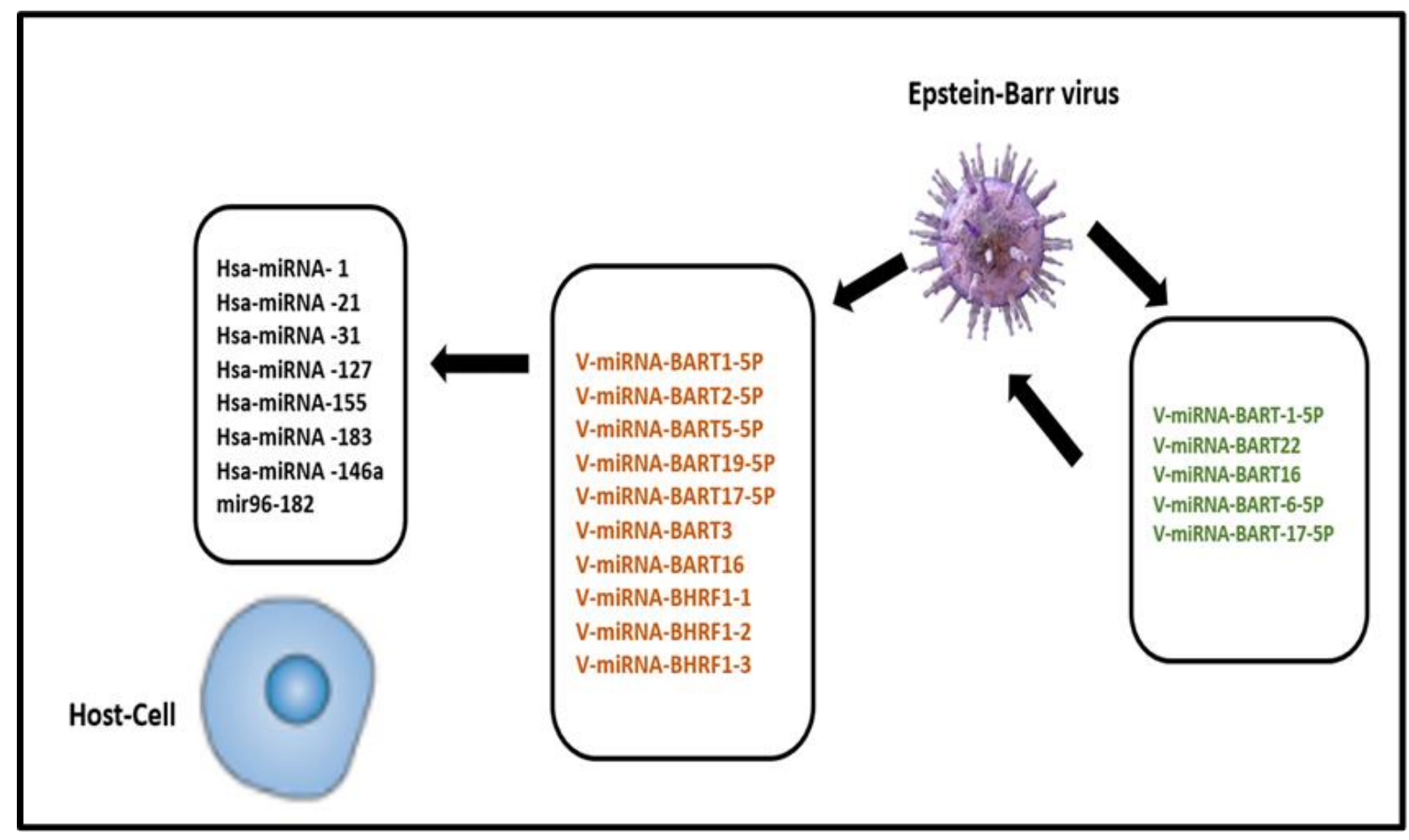

Fig 4. EBV-miRNAs and hsa-miRNAs whose expression is influenced by the viral infection. The deregulate of these miRNAs contribute to cell transformation and tumor progression. Green= miRNAs of virus which targeted viral mRNAs, brown $=$ miRNAs of virus which targeted cellular mRNAs, and yellow $=$ miRNAs of the host cell which influenced by the viral infection. 
Table 1 An overview of the EBV-miRNAs and their target genes that have been explored

\begin{tabular}{lll}
\hline \multicolumn{1}{c}{ MiRNA } & \multicolumn{1}{c}{ Target gene } & \multicolumn{1}{c}{ Related Process } \\
\hline BART and BHRF1 & EBNA1 & Latency Regulation \\
BHRF1 & PTEN & Cell Proliferation, Apoptosis \\
miR-BHRF1-1 & Not Applicable & Survival Marker in CLL \\
miR-BHRF1-2 & PRDM1 & Cell Cycle Progression, Apoptosis \\
miR-BHRF1-2-5p & IL-1 receptor & Innate Immunity \\
miR-BART6-3p & PTEN & Cell Proliferation, Apoptosis \\
& IL-6RB & Innate Immunity \\
miR-BART5-5p & RIG-I & Innate Immunity \\
miR-BART10 & PD-L1 & Apoptosis \\
miR-15-3p & B-TrCP & Cell Proliferation \\
& TAX1BP1 & anti-apoptotic TAX1BP1 \\
miR-BART7-3p & & gastric cancer (GC) cell growth \\
& PTEN & Cell growth, cell Proliferation \\
MiR-BART3 & c-Myc & cell Proliferation \\
miR-BART19-3p & c-Jun & Apoptosis \\
miR-BART5-5p & DICE1 & Apoptosis \\
miR-BART7 & WIF1 & Cell proliferation, Apoptosis \\
miR-BART22 & PD-L1 & Apoptosis \\
& TGF 1 & Cell proliferation, Apoptosis \\
miR-BART2 & MAP3K5 & Cell proliferation, Apoptosis \\
miR-BART6-5p & LMP2A & avoid host immune \\
miR-BART1-5p & BALF5 & Latency Regulation \\
\hline
\end{tabular}

\section{Mechanism of hsa-miRNA in oncogenesis}

miRNAs are short RNAs, up to 25 nucleotides in length (Hassani and Khan, 2019), and can control a variety of target genes. Various biological functions, including cell cycle, differentiation, proliferation, apoptotic stress tolerance, energy expenditure, and immune response are controlled by miRNAs (Si et al. 2019). Although onethird of all human genes are reported to be expressed by miRNAs, disordered expression of miRNAs has been reported in many types of malignancies (Vojtechova and Tachezy, 2018). About 50\% of all hsa-miRNA genes are located in fragile regions, such as small deletions, amplifications, and translocation regions, or cancer associated sites (Calin et al. 2004). miRNA encoding genes can be found on many parts of human and other animal genomes, most commonly are found on intronic part of the genome and rarely encoded on exonic part of genome (Rupaimoole et al. 2016).

Either RNA polymerase II or RNA polymerase III transcribes the miRNA gene to generate long primary miRNA (pri-miRNA) molecules (Borchert et al. 2006). In the nucleus, which consists of Drosha and DiGeorge syndrome critical region 8 (DGCR8), miRNA complexes first process a primary miRNA (pri-miRNA) into a $70 \mathrm{bp}$ precursor miRNA (pre-miRNA). The hairpin-type PremiRNA is then transported into the cytoplasm via exportin-5 and processed again through the function of Dicer to generate a mature miRNA double-strand (21-25 bp) (Shivdasani, 2006). One of the produced strands is specifically integrated into the Argonaute-containing RNA-induced silence complexes (RISC) and directly targets the mRNA. (Berkhout and Jeang, 2007) miRNAs primarily identify complementary sequences across the three UTRs of the target mRNA (Brennecke et al. 2005). The binding of miRNA to mRNA affects changes in gene expression that promote degradation or translational inhibition of mRNA. miRNAs occupy important positions in various biological systems, including proliferation, apoptotic (apoptotic) cell differentiation, etc. ( $\mathrm{Li}$ et al. 2009). They have been implicated in the development and pathogenesis of cancer (Hayes et al. 2014). The role and pathway of miRNAs in the pathogenesis of cancer remains unknown. MiRNAs are classified into two types according to their frequency and target gene, Cancerpromoting miRNAs and tumor-preventing miRNAs (Wang et al. 2019).

While miRNA biosynthesis is a controlled process, dysregulation of miRNAs induced by changes in proteins biosynthetic pathways (Drosha, Dicer and AGO2) that take place in tumor cells (Rupaimoole et al. 2016). hsamiRNA biosynthesis reveals the processes that control the expression of its target gene (Fig. 5). These changes may 
occur due to biosynthetic defect affected by hypoxia or hsa-miRNA transcriptional changes (Ma et al. 2007). Several miRNAs oncogenes are considerably raised in tumors. Pathways through which oncogenic miRNAs expression is raised in tumors are varied and miRNA specific (e.g., increased transcription of specific miRNAs) (Noman et al. 2012).

\section{Conclusion}

The virus has created a complex symbiotic system that accesses and controls the host's transcription machinery. The discovery of miRNAs has improved our knowledge of human diseases, including cancer. Recently, miRNAs have created new control components of complex gene expression pathways, affecting various disorders such as cancer. In general, the viral incubation period is the period during which most of the v-miRNA is produced, functioning as an immune evasion technique, and consequently altering the behaviour of the host to support the survival of the cell. The development of tumors induced by so-called oncoviruses must be regarded as uncommon. Cancers induced by tumor viruses occur at an even higher rate in immunosuppressed patients. It is important that EBV, which encrypts miRNAs in the initiation and development of malignant virus-related diseases, affects the expression of target genes. Accumulated data show important effects of EBVmiRNAs affecting cell proliferation, cell death (cell death), cancer and metastatic invasion and antigenic escape. The discovery of $\mathrm{v}$-miRNAs is probably a dramatic shift in our notion of host-virus interactions and their pathogenic consequences. miRNAs have the potential to be a viable approach for early detection and non-invasive treatment of viral malignancies. However, vmiRNAs, such as virus-related, are effective tools for investigating the prognosis of the disease and have important therapeutic potential for future clinical treatments and superior cancers. More research is needed to gain accurate insights into the miRNA pathway and treatment.

\section{Competing interests}

The authors declare that they have no competing interests.

\section{Funding}

No funding was used to conduct this research.

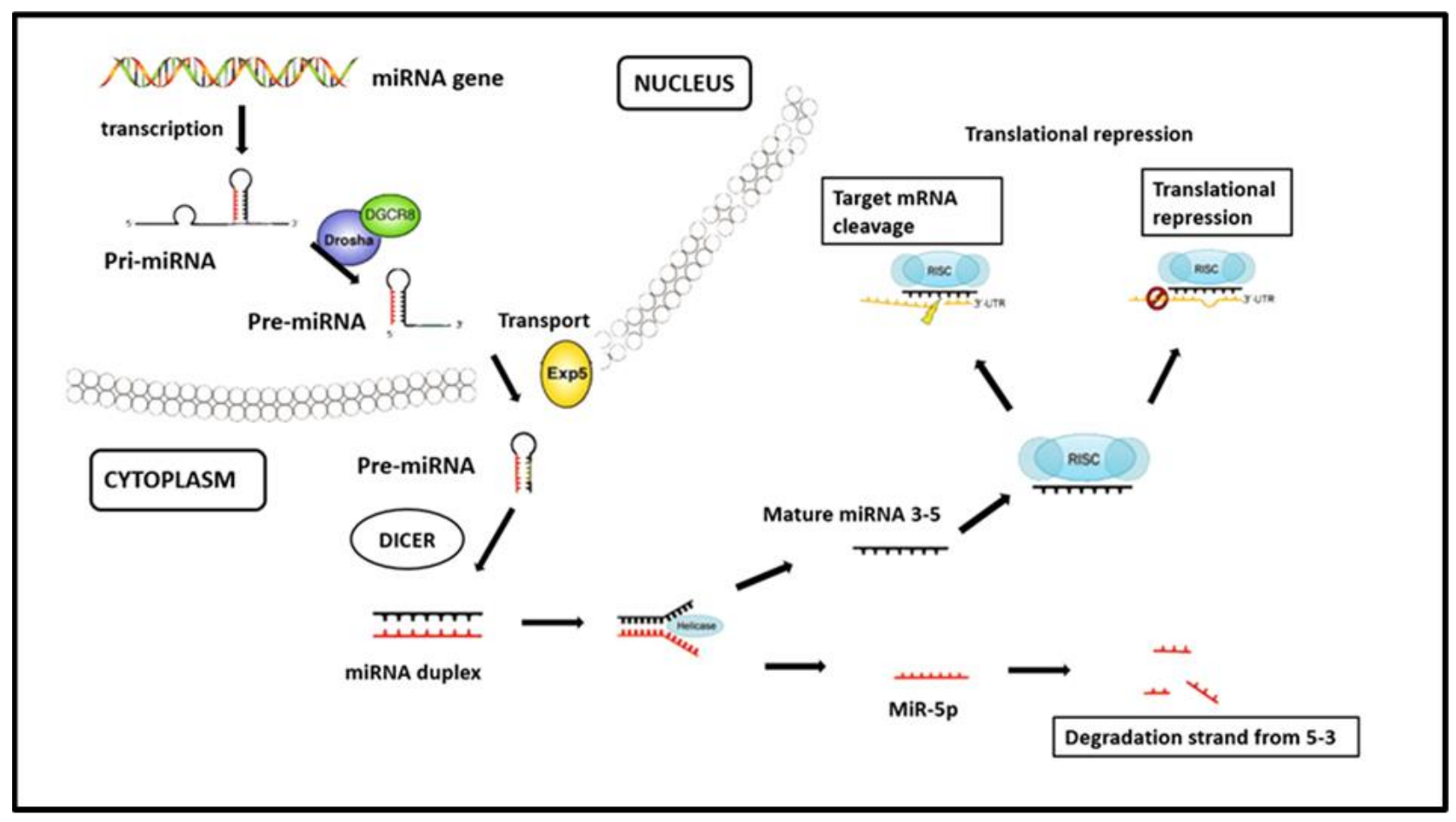

Fig 5. Model of miRNA biogenesis and posttranscriptional regulation of genes.

\section{References}


Berkhout Bm, Jeang KT (2007) RISCy business: MicroRNAs, pathogenesis, and viruses. Journal of Biological Chemistry, 282, 26641-26645.

Borchert GM, Lanier W, Davidson BL (2006) RNA polymerase III transcribes human microRNAs. Nature structural \& molecular biology, 13, 10971101.

Brennecke J, Stark A, Russell RB, Cohen SM (2005) Principles of microRNA-target recognition. PLoS biol, 3, e85.

Calin GA, Sevignani C, Dumitru CD, Hyslop T, Noch E, Yendamuri S, Shimizu M, Rattan S, Bullrich F, Negrini M, Croce CM (2004) Human microRNA genes are frequently located at fragile sites and genomic regions involved in cancers. Proc Natl Acad Sci U S A, 101, 2999-3004.

Chang Y, Moore PS, Weiss RA (2017) Human oncogenic viruses: nature and discovery. Philos Trans R Soc Lond B Biol Sci, 372.

Choy EY, Siu KL, Kok K H, Lung RW, Tsang CM, To KF, Kwong DL, Tsao SW, Jin DY (2008) An Epstein-Barr virus-encoded microRNA targets PUMA to promote host cell survival. J Exp Med, 205, 2551-60.

Cohen JI, Fauci AS, Varmus H, Nabel GJ (2011) EpsteinBarr virus: an important vaccine target for cancer prevention. Science translational medicine, 3, 107fs7-107fs7.

Cosmopoulos K, Pegtel M, Hawkins J, Moffett H, Novina C, Middeldorp J, Thorley-Lawson DA (2009) Comprehensive profiling of Epstein-Barr virus microRNAs in nasopharyngeal carcinoma. J Virol, 83, 2357-67.

Feng H, Shuda M, Chang Y, Moore PS (2008) Clonal integration of a polyomavirus in human Merkel cell carcinoma. Science, 319, 1096-100.

Gallo A, Miceli V, Bulati M, Iannolo G, Contino F, Conaldi PG (2020) Viral miRNAs as Active Players and Participants in Tumorigenesis. Cancers (Basel), 12.

Hassani A, Khan, G (2019) Epstein-Barr Virus and miRNAs: Partners in Crime in the Pathogenesis of Multiple Sclerosis? Front Immunol, 10, 695.

Hayes J, Peruzzi PP, Lawler S (2014) MicroRNAs in cancer: biomarkers, functions and therapy. Trends in molecular medicine, 20, 460-469.
Iizasa H, Wulff BE, Alla NR, Maragkakis M, Megraw M, Hatzigeorgiou A, Iwakiri, D, Takada K, Wiedmer A, Showe L, Lieberman P, Nishikura K (2010) Editing of Epstein-Barr virus-encoded BART6 microRNAs controls their dicer targeting and consequently affects viral latency. J Biol Chem, 285, 33358-33370.

Kanda T, Yajima M, Ikuta K (2019) Epstein-Barr virus strain variation and cancer. Cancer Sci, 110, 11321139.

Krump NA, You J (2018) Molecular mechanisms of viral oncogenesis in humans. Nat Rev Microbiol, 16, 684-698.

Li M, Marin-Muller C, Bharadwaj U, Chow K-H, Yao Q, Chen C (2009) MicroRNAs: control and loss of control in human physiology and disease. World journal of surgery, 33, 667-684.

Liebowitz D (1994) Nasopharyngeal carcinoma: the Epstein-Barr virus association. Seminars in oncology, 376-381.

Louten J, Beach M, Palermino K, Weeks M, Holenstein, G (2015) MicroRNAs Expressed during Viral Infection: Biomarker Potential and Therapeutic Considerations. Biomark Insights, 10, 25-52.

Ma L, Teruya-Feldstein J, Weinberg RA (2007) Tumour invasion and metastasis initiated by microRNA-10b in breast cancer. Nature, 449, 682-8.

Mclaughlin-Drubin ME, Munger K (2008) Viruses associated with human cancer. Biochimica et Biophysica Acta (BBA)-Molecular Basis of Disease, 1782, 127-150.

Morales-Sánchez A, Fuentes-Pananá EM (2014) Human viruses and cancer. Viruses, 6, 4047-79.

Noman MZ, Buart S, Romero P, Ketari S, Janji B, Mari B, Mami-Chouaib F, Chouaib S (2012) Hypoxiainducible miR-210 regulates the susceptibility of tumor cells to lysis by cytotoxic $\mathrm{T}$ cells. Cancer Res, 72, 4629-41.

Palmero EI, De Campos SG, Campos M, De Souza NC, Guerreiro ID, Carvalho AL, Marques MM (2011) Mechanisms and role of microRNA deregulation in cancer onset and progression. Genet Mol Biol, 34, 363-70.

Parkin DM (2006) the global health burden of infectionassociated cancers in the year 2002. International journal of cancer, 118, 3030-3044. 
Pavlovic A, Glavina Durdov M, Capkun V, Jakelic Pitesa J, Bozic Sakic M (2016) Classical Hodgkin Lymphoma with Positive Epstein-Barr Virus Status is Associated with More FOXP3 Regulatory $\mathrm{T}$ Cells. Med Sci Monit, 22, 2340-6.

Pfeffer S, Voinnet O (2006) Viruses, microRNAs and cancer. Oncogene, 25, 6211-9.

Pfeffer S, Zavolan M, Grässer FA, Chien M, Russo JJ, Ju J, John B, Enright AJ, Marks D, Sander C (2004) Identification of virus-encoded microRNAs. Science, 304, 734-736.

Rupaimoole R, Calin GA, Lopez-Berestein G, Sood AK (2016) miRNA Deregulation in Cancer Cells and the Tumor Microenvironment. Cancer Discov, 6, 235-46.

Rwazavian N (2011) Can a virus cause cancer: A look into the history and significance of oncoviruses. Berkeley Scientific Journal, 14.

Shivdasani RA (2006) MicroRNAs: regulators of gene expression and cell differentiation. Blood, 108, 3646-3653.

Si W, Shen J, Zheng H, Fan W (2019) The role and mechanisms of action of microRNAs in cancer drug resistance. Clin Epigenetics, 11, 25.

Tan, W., Liu, B., Qu, S., Liang, G., Luo, W. \& Gong, C. 2018. MicroRNAs and cancer: Key paradigms in molecular therapy. Oncol Lett, 15, 2735-2742.
Vojtechova Z, Tachezy R (2018) The Role of miRNAs in Virus-Mediated Oncogenesis. Int J Mol Sci, 19.

Wang M, Gu B, Chen X, Wang Y, Li P, Wang K (2019) The Function and Therapeutic Potential of EpsteinBarr Virus-Encoded MicroRNAs in Cancer. Mol Ther Nucleic Acids, 17, 657-668.

Zeng Z, Huang H, Huang L, Sun M, Yan Q, Song Y, Wei F, Bo H, Gong Z, Zeng Y, Li Q, Zhang W, Li X, Xiang B, Li X, Li Y, Xiong W, Li G (2014) Regulation network and expression profiles of Epstein-Barr virus-encoded microRNAs and their potential target host genes in nasopharyngeal carcinomas. Sci China Life Sci, 57, 315-326.

Zhou L, Bu Y, Liang Y, Zhang F, Zhang H, Li S (2016) Epstein-Barr Virus (EBV)-BamHI-A Rightward Transcript (BART)-6 and Cellular MicroRNA-142 Synergistically Compromise Immune Defense of Host Cells in EBV-Positive Burkitt Lymphoma. Med Sci Monit, 22, 4114-4120.

Židovec Lepej S, Matulić M, Gršković P, Pavlica M, Radmanić L, Korać P (2020) miRNAs: EBV Mechanism for Escaping Host's Immune Response and Supporting Tumorigenesis. Pathogens, 9. 